\title{
A Spin Polarized Disc
}

\author{
J. P. Krisch ${ }^{1}$ and L. S. Smalley ${ }^{2}$
}

Received June 21, 1994

\begin{abstract}
We present a solution to the gravitational field equations in a RiemannCartan spacetime. The solution describes a disc of infinite radius and finite thickness. The solution has three forms which depend on the size of the acceleration. The matter content of the disc is a rotating spin fluid with a constant $z$ acceleration and a spin density polarized along the axis of rotation. The fluid has zero axial and tangential pressures. There is a radial pressure. The energy density and pressure are finite within the disc.
\end{abstract}

\section{INTRODUCTION}

The Van Stockum [1] dust solution describes an infinite cylinder of unaccelerated dust rotating about the $z$-axis. The parameters of the model are the angular velocity, the radius of the cylinder, and the zero acceleration. This solution, while probably not physical, has provided many useful insights into general relativistic solutions. One of the very interesting things about the Van Stockum solution is the existence of three exterior vacuum matches to the stationary dust interior [2]. Which exterior solution is chosen depends on the mass per unit length of the cylinder.

In this note we describe an infinite disc solution that is similar to the Van Stockum infinite cylinder. It is a radially infinite disc of finite thickness containing matter with constant $z$-acceleration. The matter content

1 Department of Physics, The University of Michigan, Ann Arbor, Michigan 48109, USA

2 Department of Physics, University of Alabama in Hunstville, Huntsville, Alabama 35899, USA 
of the disc is an axially polarized spin-fluid in a Riemann-Cartan spacetime. The fluid is rotating about the $z$-axis. There is zero pressure in the axial and tangential directions. A non-zero finite radial pressure exists. The energy density of the disc is positive. For a fixed $g_{z z}$, the parameters of the solution are the thickness of the disc, the constant $z$-acceleration, the divergence of the spin density and the angular velocity. There is a fluid singular surface which may be avoided by properly limiting the disc thickness. As in the Van Stockum exterior, we find that there are three possible interior parameter ranges, depending on the deviation of the thickness from the classical value.

Cylindrically symmetric solutions with isotropic pressures have been discussed by Krasiński [3]. In other calculations concemed only with the surface stress energy content of the source, discs have been used frequently [4-7]. The solution presented in this note has a well-defined and simple, if anisotropic, interior stress energy content. The solution may be useful in modeling large rotating structures with embedded intrinsic angular momentum.

\section{FIELD EQUATIONS}

\subsection{Metric and matter content.}

We wish to consider the field equations for a Riemann-Cartan spacetime with metric

$$
\begin{aligned}
d s^{2}=-f d t^{2}-2 k d \phi & d t+\exp (\mu) d r^{2}+\exp (\beta) d z^{2}+\frac{D^{2}-k^{2}}{f} d \phi^{2}, \\
f & =\left(r+r_{0}\right)^{d} F(z)=\left(r+r_{0}\right)^{d} \exp [2 a z] \\
k & =\left(r+r_{0}\right)^{g} G(z) \\
D & =\left(r+r_{0}\right)^{s} D(z) \\
\exp (\mu) & =\exp [\mu(z)] \\
\exp (\beta) & =\left(r+r_{0}\right)^{b} \exp [-2 B z] .
\end{aligned}
$$

The constant $z$-component fluid acceleration,

$$
\frac{F(z)}{2 F(z)}=a
$$

has been imposed (prime (') is used to denote differentiation with respect 
to $z$ ). It is convenient to introduce the tetrads that diagonalize the metric,

$$
\begin{aligned}
& e_{(0)}^{\nu}=\left(\frac{1}{\sqrt{f}}, 0,0,0\right) \\
& e_{(1)}^{\nu}=\left(0, \exp \left[\frac{-\mu(z)}{2}\right], 0,0\right) \\
& e_{(2)}^{\nu}=\left(0,0, \exp \left[\frac{-\beta(z)}{2}\right], 0\right) \\
& e_{(3)}^{\nu}=\left(\frac{-k}{\sqrt{f} D}, 0,0, \frac{\sqrt{f}}{D}\right) .
\end{aligned}
$$

These tetrads will be used to index the field equations.

The spacetime contains a spin fluid - a perfect fluid with a spin density, $S_{i j}$, defined throughout its extent. The stress energy tensor for this kind of fluid has been developed and discussed extensively by Ray and Smalley [8]. Using cylindrical coordinates $(r, \phi, z)$ we have three possible spin densities $S_{r \phi}, S_{z \phi}, S_{r z}$. We wish to develop a solution describing a disc with a axially polarized spin content; $S_{r \phi}$ will be the only non-zero spin density. $S_{r z}$ is non zero only if $f$ and $k$ are directly proportional, i.e. the fluid is completely irrotational in general relativity. We will avoid this case. Zeroing $S_{z \phi}$ will constrain the metric potentials.

2.2. Spin-metric contraints. The off diagonal field equations.

We will focus on the particular case where the spin densities $S_{r \phi}$ and $S_{z \phi}$ are proportional to the classical fluid vorticities.

$$
\begin{aligned}
& S_{r \phi}=\frac{f k_{r}-k f_{r}}{f \sqrt{f}}=\frac{k}{\sqrt{f}} \frac{(g-d)}{r+r_{0}}, \\
& S_{z \phi}=\frac{f k_{z}-k f_{z}}{f \sqrt{f}}=\frac{-k}{\sqrt{f}}\left(\frac{F^{\prime}}{F}-\frac{G^{\prime}}{G}\right) .
\end{aligned}
$$

Requiring $S_{z \phi}$ to be zero imposes the constraint

$$
\begin{aligned}
\frac{F^{\prime}}{F} & =\frac{G^{\prime}}{G}=2 a \\
G(z) & =c_{1} F(z)=c_{1} \exp [2 a z]
\end{aligned}
$$

with $c_{1}$ an integration constant.

Requiring the spin density to be proportional to the vorticity creates a special type of solution to the field equations in an RC space time. It 
has been shown that with the relations (5),(6), the diagonal and $r z$ field equations are formally identical to the field equations for a static general relativistic perfect fluid [9] The remaining (03) field equation is used to determine the off diagonal metric component in the RC spacetime. The field equation that constrains the metric potentials is the (tetrad indexed) (03) equation,

$$
\begin{aligned}
& \left(r+r_{0}\right)^{(b-2)}(d-g) d \\
& \quad=\frac{F^{\prime}(z)}{F(z)}\left(\frac{F^{\prime}(z)}{F(z)}-\frac{G^{\prime}(z)}{G(z)}\right) \exp [\mu(z)+2 B z] .
\end{aligned}
$$

Requiring $S_{z \phi}$ to be zero and $S_{r \phi}$ non zero implies that

$$
d=0 \text {. }
$$

The $r z$ field equation provides further constraining relations on the metric functions.

$$
(r z): \quad \mu^{\prime}(z) s+(b-2 s) \frac{D^{\prime}}{D}+s \frac{F^{\prime}}{F}=0 .
$$

There are three possibilities:

$$
\begin{aligned}
& \text { (a) } s=0, \quad b=0, \\
& \text { (b) } s=0, \quad D^{\prime}=O, \\
& \text { (c) } s \neq 0, \quad \mu^{\prime}(z)+\frac{F^{\prime}}{F}+\frac{b-2 s}{s} \frac{D^{\prime}}{D}=0 .
\end{aligned}
$$

Case (b) produces unphysical fluid parameters. The infinite disc solutions belong to the parameter set $(s=b=d=0, g \neq 0)$. There are some interesting solutions in case (c) which will be described elsewhere.

The metric that we are considering can now be written as

$$
\begin{aligned}
d s^{2}= & -\exp [2 a z]\left(d t+c_{1}\left(r+r_{0}\right)^{g} d \phi\right)^{2}+\exp [\mu(z)] d r^{2} \\
& +\exp [-2 B z] d z^{2}+D^{2}(z) \exp [-2 a z] d \phi^{2}
\end{aligned}
$$

We will impose the axial and tangential dust condition in the next section. 2.3. Fluid parameters. The diagonal field equations. 
The diagonal field equations generate the pressure and energy density. For case (a) the (tetrad indexed) diagonal stress-energies are

$$
\begin{aligned}
p_{1}= & \exp [2 B z]\left(\frac{D^{\prime \prime}}{D}+B \frac{D^{\prime}}{D}+a^{2}-a \frac{D^{\prime}}{D}\right) \\
p_{2}= & \exp [2 B z]\left(-a^{2}+a \frac{D^{\prime}}{D}+\frac{\mu^{\prime} D^{\prime}}{2 D}\right)=0 \\
p_{3}= & \exp [2 B z]\left(a^{2}+\left(\frac{\mu^{\prime}+2 B}{4}\right)\left(2 a+\mu^{\prime}\right)+\frac{\mu^{\prime \prime}}{2}\right)=0 \\
\epsilon= & \exp [2 B z]\left(-\frac{D^{\prime \prime}}{D}-\frac{\mu^{\prime \prime}}{2}+2 a \frac{D^{\prime}}{D}\right. \\
& \left.+\frac{\mu^{\prime}+2 B}{4}\left(2 a-\frac{2 D^{\prime}}{D}-\mu^{\prime}\right)-a^{2}\right)
\end{aligned}
$$

\section{THE SOLUTIONS}

\subsection{Metric solutions.}

Equations (16) and (17) provide two constraints. Equation (16) will determine $D(z)$ as a function of $\mu(z)$.

$$
\frac{D^{\prime}}{D}=\frac{2 a^{2}}{\mu^{\prime}+2 a}
$$

Equation (17) becomes

$$
2 a^{2}+\left(\frac{\mu^{\prime}+2 B}{2}\right)\left(2 a+\mu^{\prime}\right)+\mu^{\prime \prime}=0 .
$$

The solutions of eq. (20) provide the three different fluid descriptions. Equation (20) can be rewritten as

$$
\int \frac{-d \mu^{\prime}}{\left(\mu^{\prime}+a+B\right)^{2}+4 a^{2}-(a-B)^{2}}=\frac{z}{2}-z_{0} .
$$

The three different solutions depend on the value of $S$ where

$$
S^{2}=4 a^{2}-(a-B)^{2},
$$

Solution 1: $S^{2}>0$,

Solution 2: $S^{2}=0, \quad$ (a) $B=3 a$,

(b) $B=-a$

Solution 3: $\quad-Y^{2}=S^{2}<0$. 
In investigating the solution structure, constant scale factors will be absorbed into the coordinates.

Solution 1: $u_{1}=S\left(z_{0}-z / 2\right), S^{2}=(3 a-B)(a+B)>0$.

Integrating (21) one finds

$$
\begin{aligned}
& \exp (\mu(z))=\cos ^{2}\left(u_{1}\right) \exp [-(a+B) z] \\
& D(z)=\frac{\exp [(a-B) z / 2]}{(a-B) \cos \left(u_{1}\right)+S \sin \left(u_{1}\right)} .
\end{aligned}
$$

The fluid parameters for this case are

$$
\begin{aligned}
p & =\exp [2 B z] \frac{2 a^{2} S^{2}}{\left[(a-B) \cos \left(u_{1}\right)+S \sin \left(u_{1}\right)\right]^{2}}, \\
e & =\frac{p}{2 a}\left(S \tan \left(u_{1}\right)-a-B\right) .
\end{aligned}
$$

Solution 2: $u_{2}=z-2 z_{0},(3 a-B)(a+B)=0$.

Integrating (21) one finds

$$
\exp [\mu(z)]=\left(z-2 z_{0}\right)^{2} \exp [-z(B+a)]
$$

and

$$
D(z)=\exp \left[2 a^{2} z /(a-B)\right]\left\{(a-B)\left(z-2 z_{0}\right)+2\right\}^{w}
$$

with $w=-[2 a /(a-B)]^{2}$.

The fluid parameters are

$$
\begin{aligned}
p & =\frac{8 a^{2} \exp [2 B z]}{\left(2+(a-B)\left(z-2 z_{0}\right)\right)^{2}} \\
e & =\frac{p}{2 a}\left[\frac{2-(a+b) u_{2}}{u_{2}}\right.
\end{aligned}
$$

For case $2 \mathrm{a}, B=3 a$, we have

$$
p=\frac{2 a^{2} \exp [6 a z]}{\left(1-a\left(z-2 z_{0}\right)\right)^{2}}, \quad e=p \frac{\left(1-2 a\left(z-2 z_{0}\right)\right)}{a\left(z-2 z_{0}\right)}
$$

and for case $2 \mathrm{~b}, B=-a$ we have

$$
p=\frac{2 a^{2} \exp [-2 a z]}{\left(1+a\left(z-2 z_{0}\right)\right)^{2}}, \quad e=p \frac{1}{a\left(z-2 z_{0}\right)} .
$$


Solution 3: $u_{3}=Y\left(-z_{0}+z / 2\right), Y^{2}=(B-3 a)(a+B)>0$. Integrating (21) one finds

$$
\exp [\mu(z)]=\exp [-(a+B) z] \cosh ^{2}\left(u_{3}\right)
$$

and

$$
D=\frac{\exp [(a-B) z / 2]}{Y \sinh \left(u_{3}\right)+(a-B) \cosh \left(u_{3}\right)} .
$$

The fluid parameters are

$$
\begin{aligned}
p & =\exp [2 B z] \frac{-2 a^{2} Y^{2}}{\left[(a-B) \cosh \left(u_{3}\right)+Y \sinh \left(u_{3}\right)\right]^{2}}, \\
e & =\frac{p}{2 a}\left[Y \tanh \left(u_{3}\right)-a-B\right] .
\end{aligned}
$$

Note that the parameter choice $B=0, a \neq 0, a$ real, will be described by solution 1 . For all of the solutions the spin density is

$$
S_{r \phi}=\frac{k_{r}}{\sqrt{f}}=c_{1} \exp [a z] g\left(r+r_{0}\right)^{g-1} .
$$

This is also the structure of the vorticity. The choice of the parameter $g$, the radial exponent in $g_{0 \phi}$ has physical implications for the fluid. The non zero component of the spin density divergence in the RC spacetime is

$$
W_{\phi}=c_{1} g(g-1)\left(r+r_{0}\right)^{g-2} \exp [a z] \exp [-\mu(z)] .
$$

$g=1$ describes a divergence free spin density.

\section{THE DISC THICKNESS}

\subsection{The Boundary points.}

Limitations on the positivity and finiteness of the energy density define the range of the $z$ coordinate which can, in turn, be related to the thickness of the disc. The calculation is similar for all of the solutions except $2 \mathrm{~b}$. It is not possible to define an upper boundary and therefore a disc for this solution. We will use solution $2 \mathrm{a}$ as an example. From eq. (31), the energy density for this case is

$$
e=\frac{2 a^{2} \exp [6 a z]}{\left(1-a\left(z-2 z_{0}\right)\right)^{2}} \frac{\left(1-2 a\left(z-2 z_{0}\right)\right)}{a\left(z-2 z_{0}\right)} .
$$


For this energy density to be positive or zero requires

$$
z \leq \frac{1}{2 a}+2 z_{0} \text {. }
$$

This limit will also keep the pressure well behaved. We will take the equal sign to define the upper boundary of the disc

$$
z_{\max }=H=\frac{1}{2 a}+2 z_{0} .
$$

Note that this inmplies a zero energy density over the upper boundary. By introducing a new parameter it would be possible to keep the upper boundary under this point, thus providing a non zero upper boumdary energy. We do not do this for the upper boundary but will for the lower boundary point. To keep the energy finite we require

$$
z>2 z_{0} \text {. }
$$

To set the lower boundary we introduce a parameter $\delta$ and define

$$
z_{\min }=-H=\frac{2 z_{0}}{\delta}, \quad 0<\delta<1 .
$$

Using (39) and (41) we can write $H$ in terms of $\delta$,

$$
H=\frac{1}{2 a(1+\delta)} \text {. }
$$

$\delta$ is a parameter that will replace $z_{0}$ and can be used in modeling calculations to vary the boundary position for a fixed acceleration.

\subsection{The thickness.}

The proper thickness of the disc is

$$
T=\int_{-H}^{H} \exp (-B z) d z=2 H \frac{\sinh (B H)}{B H} .
$$

The three solution regions correspond to the passage from the classical disc thickness to the relativistic thickness. Assuming positive acceleration, for $B \ll 3 a$ we have $T=2 H$, the euclidian value. In the transition region of the second solution, $2 a$, we can examine the range of thickness as we vary $H$ for a fixed acceleration. Letting $\delta$ run from 0 to 1 we have

$$
1.1<\frac{T}{2 H}<1.42 \text {. }
$$

Solution 3 corresponds to a thickness much larger than the classical value. It is very interesting that this classification is made by comparison with an acceleration rather than an angular velocity as in the Van Stockum case. 


\section{CONCLUSION}

In conclusion, we have presented a solution describing a spin polarized disc of fluid in a Riemann-Cartan spacetime. The disc could possibly be used to model a large rotating structure. As an example, using solution 2, we estimate the acceleration, and central energy density of a disc of proper thickness $T=n$ light years $=9.46 n \times 10^{15} \mathrm{~m}$. We use $\delta=\frac{1}{2}$. From (44) we have $a H=\frac{1}{3}$ and from (45)

$$
a=\frac{8.3}{n} 10^{-17} \mathrm{~m}^{-1}
$$

and

$$
e=\frac{2 a^{2}(1-2 a H \delta)}{(1-a H \delta)^{2}(a H \delta)}=\frac{7.9 \times 10^{-32}}{n^{2}} \mathrm{~m}^{-2}
$$

which, for $n$ about 1 , is of the order of $10^{12}$ joules $/ \mathrm{m}^{3}$. A limitation of the model for small scale modeling is, of course, the lack of a radial fall off. However, like the Van Stockum infinite cylinder, this simple infinite disc may prove to be an interesting and useful solution.

\section{REFERENCES}

1. Van Stockum, W. (1937). Proc. Roy. Soc. Edinburgh 57, 135.

2. Bonner, W. B. (1980). J. Phys. A13, 2121.

3. Krasiński, A. (1975). Acta Phys. Polon. B6, 223.

4. Israel, W. (1966). Nuovo Cimento XLIV,, 1.

5. Israel, W. (1970). Phys. Rev. D2, 641.

6. Hamity, V. H., and Lamberti, P. W. (1987). Gen. Rel. Grav. 19, 917; Lamberti, P. W., and Hamity, V. H. (1989). Gen. Rel. Grav. $21,869$.

7. Bičak, J., and Ledvinka, T. (1993). Phys. Rev. Lett. 11, 1669.

8. Ray, J., and Smalley, L. S. (1982). Phys. Rev. Lett. 49, 1059; 50, 626(E); (1983). Phys. Rev. D27, 1383.

9. Krisch, J., and Smalley, L. S. (1993). Class. Quant. Grav. 10, 995. 